\title{
BMJ Open Impact on health and provision of healthcare services during the COVID-19 lockdown in India: a multicentre cross-sectional study
}

\author{
Rajiv Raman (1) , ${ }^{1}$ Ramachandran Rajalakshmi, ${ }^{2}$ Janani Surya, ${ }^{3}$ \\ Radha Ramakrishnan, ${ }^{4}$ Sobha Sivaprasad, ${ }^{5}$ Dolores Conroy, ${ }^{4}$ Jitendra Pal Thethi, ${ }^{6}$ \\ V Mohan, ${ }^{7}$ Gopalakrishnan Netuveli ${ }^{8}$
}

To cite: Raman $R$,

Rajalakshmi R, Surya J, et al. Impact on health and provision of healthcare services during the COVID-19 lockdown in India: a multicentre crosssectional study. BMJ Open 2021;11:e043590. doi:10.1136/ bmjopen-2020-043590

- Prepublication history and supplemental materials for this paper is available online. To view these files, please visit the journal online (http://dx.doi org/10.1136/bmjopen-2020043590).

Received 10 August 2020 Revised 28 December 2020 Accepted 04 January 2021

Check for updates

(C) Author(s) (or their employer(s)) 2021. Re-use permitted under CC BY-NC. No commercial re-use. See rights and permissions. Published by BMJ.

For numbered affiliations see end of article.

Correspondence to

Dr Rajiv Raman;

rajivpgraman@gmail.com

\section{ABSTRACT}

Introduction The COVID-19 pandemic resulted in a national lockdown in India from midnight on 25 March 2020, with conditional relaxation by phases and zones from 20 April. We evaluated the impact of the lockdown in terms of healthcare provisions, physical health, mental health and social well-being within a multicentre crosssectional study in India.

Methods The SMART India study is an ongoing house-tohouse survey conducted across 20 regions including 11 states and 1 union territory in India to study diabetes and its complications in the community. During the lockdown, we developed an online questionnaire and delivered it in English and seven popular Indian languages (Hindi, Tamil, Marathi, Telegu, Kannada, Bengali, Malayalam) to random samples of SMART-India participants in two rounds from 5 May 2020 to 24 May 2020. We used multivariable logistic regression to evaluate the overall impact on health and healthcare provision in phases 3 and 4 of lockdown in red and non-red zones and their interactions.

Results A total of 2003 participants completed this multicentre survey. The bivariate relationships between the outcomes and lockdown showed significant negative associations. In the multivariable analyses, the interactions between the red zones and lockdown showed that all five dimensions of healthcare provision were negatively affected (non-affordability: OR $1.917(95 \% \mathrm{Cl} 1.126$ to 3.264), non-accessibility: OR 2.458 (95\% Cl 1.549 to 3.902), inadequacy: OR 3.015 (95\% Cl 1.616 to 5.625$)$, inappropriateness: OR $2.225(95 \% \mathrm{Cl} 1.200$ to 4.126$)$ and discontinuity of care: OR 6.756 (95\% Cl 3.79 to 12.042)) and associated depression and social loneliness.

Conclusion The impact of COVID-19 pandemic and lockdown on health and healthcare was negative. The exaggeration of income inequality during lockdown can be expected to extend the negative impacts beyond the lockdown.

\section{INTRODUCTION}

Social distancing is a successful mitigation strategy used to slow transmission rates during communicable disease outbreaks. ${ }^{1}$ Unlike other virus epidemics, COVID-19, caused by SARS-CoV-2, rapidly evolved into a
Strength and limitations of this study

- This multicentre study highlights the impact of the lockdown on healthcare services and general, social and mental health of the population.

- We were able to study the impact based on the severity of lockdown by comparing phases 3 and 4 of the lockdown and also differences on impact in red and non-red zones and influence of lockdown in people with diabetes.

- The interactions between the pandemic and lockdown have resulted in both positive and negative impacts.

- As this is a cross-sectional study and data were not collected prior to lockdown, we are unable to infer causality.

pandemic and it was declared a global health emergency by WHO on 30 January $2020 .^{2}$ Preventive strategies to mitigate the mortality rates due to this virus have been challenging even in many high-income countries with low population density. ${ }^{3}$

The government of India declared a nationwide lockdown from 25 March 2020 as a preventative measure against the COVID-19 pandemic in India when the numbers of recorded cases were only 519, with nine reported deaths. ${ }^{4}$ Although there was significant scepticism about this world's largest lockdown, maintaining social distancing in India is a mammoth challenge. Living conditions vary widely in India, population density range from an average of 1202-54000 people per square mile in cities such as Mumbai, and India is home to $17 \%$ of the global population. Given the risks that India faced if COVID-19 affected the nation at the pace that it affected Italy and other countries, the government of India had to rapidly respond with such a strategic decision. 
As population movement from outside and within India maintained the rise of cases, ${ }^{5}$ the lockdown had to be extended further on three occasions, referred to as lockdown phases 1-4 and conditional relaxation of the lockdown is still ongoing. As there were regional differences in COVID-19 cases, the 739 districts in India (average population $1.7 \times 10^{6}$ but can range from $57 \times 10^{3}$ to $4 \times 10^{6}$ ) were also divided into zones colour coded as red, orange and green during phases $2-4$ based on the doubling rates, with the red zone representing the highest doubling rates.

There was global press coverage on this lockdown related disruption, chaos, financial difficulties, lack of shelter and food for the vulnerable populations, fear, social exclusion, stigma and misinformation on COVID19. However, there is a lack of evidence of the impact of this lockdown on the health of individuals at population level.

Given that $70 \%$ of the healthcare in India is provided by the private sector and most only provided minimal services during the lockdown, we hypothesised that the immediate consequences of lockdown would be restricted healthcare provisions and negative impact on the general health, mental health and social well-being of the population. ${ }^{6}$ Reduced capacity due to social distancing and other restrictions after relaxation may also be a cause of interruptions to healthcare provision.

We report here the results of a questionnaire-based survey designed to study the impact on healthcare provisions and general, social and mental health of individuals that participated in a multicentre study (SMART India). ${ }^{7}$ Our research question was 'Will the severity of the pandemic and restrictiveness of lockdown stage affect the access to healthcare and the health and well-being of the participants in the SMART India study?'

\section{METHODS}

All participants gave verbal informed consent before data collection for this survey.

\section{Smart India study}

The SMART India study, funded by the Global Challenge Research Fund and UK Research \& Innovation (UKRI), is a household survey on diabetes and its complications. The aim of the SMART India study is to develop practical and affordable models to (1) diagnose people with diabetes and pre-diabetes and (2) identify those at risk of diabetes complications so that these models can be applied to the population in low-income and middle-income countries (LMIC) where laboratory tests are unaffordable. ${ }^{7}$

The study sample was based on a multistage, stratified sampling technique using data from the 2011 census of India and covered different geographical regions (North, South, East and West) and included 11 states and 1 union territory: Assam, Chhattisgarh, Karnataka, Kerala, Madhya Pradesh, Uttar Pradesh, Maharashtra, Odisha, Tamil Nadu, Telangana, West Bengal and Delhi. All survey clusters were independent samples. Each region had three clusters stratified into urban, rural and a pre-defined special category of population such as people with poor access to healthcare, or cohorts that are presumed high risk or low risk of developing diabetes. Each cluster will screen 800-2000 consenting participants 40 years or older for a cumulative sample size of a minimum of 48000 .

In each region, a census enumeration block that usually consists of 125-150 households with a population of 650-700 was the primary sampling unit for urban areas while villages were defined in the rural areas. Bigger villages were further divided to ensure that approximately 300 households could be covered. The house-to-house survey was conducted by approaching each household in consecutive streets in each area.

The inclusion criteria are adults who are $\geq 40$ years of age (special groups may contain adult population of any age) who are local residents of Indian origin and are willing to give informed consent. Exclusion criteria include vulnerable adults in whom it may not be possible to carry out all the tests; pregnant and breast feeding women; anyone in the opinion of the fields worker deemed too ill to be screened; and those who are currently participating in intervention trials with investigational medicinal products.

In the SMART India study, diabetes was defined as known diabetes on anti-diabetes medications or diet controlled or a random blood sugar of $>200 \mathrm{mg} / \mathrm{mL}$ $(8.9 \mathrm{mmol} / \mathrm{mmol})$.

\section{Participants for the COVID-19 questionnaire study}

Two random samples in phases 3 and 4 of the lockdown were obtained from participants of the ongoing SMART India study. ${ }^{8}$ First sample was a random sample of the whole SMART India cohort and second random sample included people with diabetes from the study cohort to ensure we had a good proportion of the sample consisting of people who would need to access healthcare. We analysed both samples together because of the low cell counts when adjusting for the complex design relatively large numbers of variables. Weighting was used to ensure the age and gender distribution of our samples were similar to that of the latest census of India.

\section{Severity of lockdown in India and description of zones}

The lockdown in India was divided into phases. During phase 1 of the lockdown, people were urged to stay indoors, all services, except healthcare and other essential services, were closed, non-essential transport was suspended and most activities were prohibited. In phase 2 , the country was divided into three zones: red, orange and green based on the number of cases of COVID-19 and spread of the virus. In red zones, all forms of public transport, for example, by air, rail, road were suspended, most institutions were closed, and gatherings of people for any purpose was prohibited. Only emergency services and those related to healthcare were allowed. In orange zones, outpatient departments and clinics were allowed to be open with social distancing norms and other 
safety precautions. Limited movements of taxis and cabaggregators were allowed, and interdistrict movement of people for permitted activities was allowed. In the green zones, most restrictions were removed in a phased manner.

In phase 3 , the red zone districts continued to remain under lockdown. The orange zone districts had no public transportation but private and hired vehicles were permitted and green zone districts operated with buses limited to $50 \%$ capacity. In phase 4 , the states were given more power in the implementation of lockdown and delineation of the zones. Our COVID-19 lockdown study was conducted during phases 3 and 4 of the lockdown.

Four lockdown stages can be described in the order of strictness of lockdown from the least to the most: phase 3 lockdown-non-red zones, phase 3 lockdown-red zones, phase 4 lockdown-non-red zones and phase 4 lockdown-red zones.

\section{Questionnaire}

The questionnaire was designed to evaluate provision of healthcare services and self-rated impact of COVID-19 lockdown on physical, mental and social well-being and was developed from existing validated instruments from Europe. It was translated to seven different languages to address non-English-speaking population. Accuracy of the translations was checked by three health professionals per language. Prior to the survey being carried out a test-run was done on 14 participants, who were not part of the SMART-India study, to cross-validate the questionnaire. The fieldworkers from the 20 centres in the SMART India study were trained to administer the questionnaire by telephone and the data were entered into an electronic cloud-based database, developed specifically for the SMART India study. The data management group consisted of two project coordinators and an IT expert. A checklist was prepared and weekly web meetings conducted with the interviewers. In each call, two interview forms were randomly picked and checked for accuracies. The data management group monitored, checked and verified the data entry before the database was locked for analysis.

\section{Outcome variables}

The outcomes were the five dimensions of healthcare provision viz. availability, affordability, accessibility, appropriateness, and adequacy, patient experience and continuity of care and measures of self-reported physical health, depression, emotional and/or social loneliness. For this analysis, we derived binary variables describing poor or negative outcomes using a subset of questions.

\section{Dimensions of healthcare provision}

We measured three aspects of healthcare services, that is, access, patient experience and continuity of care. Access was defined as 'a general concept that summarises a set of more specific dimensions describing the fit between the patient and the healthcare system. ${ }^{, 9}$ Five inter-related but distinct dimensions, 5 'A's, were defined for access: adequate, accessible, affordable, appropriate and available. ${ }^{10}$ We adapted questions from a patient-led survey developed by European Patients Forum to measure access. ${ }^{11}$

Availability of healthcare was measured using a question about ease of obtaining overall access and scored 1 if accessing information was not easy. Affordability was measured by asking whether the participant experienced financial difficulties due to spending on healthcare during the lockdown ( $1=$ sometimes or often). Accessibility of healthcare was measured by counting the number of times there was delay in getting services and dichotomised as none vs one or more. Similarly, adequacy of healthcare was measured by three statements on provision of information on treatment options, adapting treatment to changing needs and involvement in the treatment decisions. We scored 1 if the response to any of the questions was 'No'. Finally, appropriateness was measured using a question on whether the participant was treated for their medical condition differently to that they received prior to COVID-19 (1=treated differently).

We then measured the patient experience of healthcare during this time, which we measured by adapting the communication section of a questionnaire developed for LMIC. ${ }^{12}$ We asked five questions on whether on approaching healthcare providers, the participants were treated with courtesy and respect, were listened to and received understandable information. We scored one if the response to any of these questions was 'false'. Lastly, we assessed whether the quality of care patients received during the lockdown is a break in the continuity of care. We used the concept of 'experienced continuity of care ${ }^{, 13}$ and measured it using four questions based on four dimensions, longitudinal, relational, flexible and cross-boundary. ${ }^{14}$ The binary variable was scored 1 if the response to any of these questions was 'No'.

\section{Measures of self-reported health impacts of COVID-19}

The impact of the lockdown on individuals was measured on physical health, mental health and social well-being. Physical health was measured using a single-item comparative self-rating question asking the participants 'compared with before the lock down, how is your health in general?' with a 5-point Likert scale response ranging from 'very good' to 'very poor' and dichotomised as poor heath for responses fair to very poor vs good and very good. Mental health was measured using the WHO-5 well-being index. ${ }^{15}$ The responses were noted on a 6-point Likert scale scored 0 ('at no time') to 5 ('all of the time') with the scores converted to a percentage scale. We dichotomised the score at the cut-off $<51$ to create a binary variable labelled 'Screening diagnosis of depression'.

To measure loneliness, we used the short De Jong Gierveld Loneliness scale ${ }^{16}$ consisting of six Likert scale items with five levels: 'none of the times', 'rarely', 'sometimes', 'often' and 'all of the time'. Three of these items measured emotional loneliness referring to a sense of 
emptiness, missing people around oneself and a feeling of rejection. The other three items referred to social loneliness referring to a lack of boarder engagement with social networks. We recoded the three items of social loneliness ${ }^{17}$ so that for all items the higher score meant greater loneliness. The scale was further dichotomised so that 'none of the times' and 'rarely' counted as zero and the rest as one. The two sub scales ranged from 0 to 3 and the total loneliness score ranged from 0 to 6 . The subscales were summed only if all items were non-missing while in computing the total score at most one missing item among the six can be accommodated. The subscales and the total score were dichotomised at $>1$ to produce binary variables representing emotionally lonely, socially lonely and lonely.

\section{Statistical analysis}

Sample size

In the absence of any pre-existing data, we assumed that a modest $50 \%$ of the participants will access healthcare services and during the lockdown there will be a $10 \%$ reduction in the proportion participants accessing healthcare services. Based on that we computed that to identify that difference with $80 \%$ power and at a 0.05 significance level, we will need 609 participants.

\section{Analysis}

The outcomes were each dimension of healthcare provision and measures of self-reported physical health, depression, emotional and/or social loneliness. Our primary exposure variables were zones of lockdown (red or not red) and phase of lockdown (phase 3 or phase 4). Control variables in the analyses included age, gender, rural vs urban residents, self-rated financial difficulties (none, some or great), occupation (working or not), financial situation (difficulty in meeting daily needsnone, some or great) and living arrangements (large household, with spouse, alone or living with unrelated individuals). In analysing this data, we took into account the stratification in the data by multiple centres and used population weights by using svy commands with STATA (V.15). We created weights based on age and gender distribution of the Indian population. The target population in our survey was between 25 and 90 years and the weights align our sample with the latest Indian Census (2011) (online supplemental table S1).

Multiple logistic regression models were used to estimate the associations between outcomes and exposures. In all models, we included an interaction term between red zone and lockdown stage. Other interactions such as between zones, stage and presence of diabetes were tested but were not significant and not used in the final models tested. The analyses took into account stratification by centres and population weights.

After a reviewer had suggested that our sample size might lack sufficient power, we estimated post hoc power through simulation. For this, we drew 10000 random samples with replacement with the same size as our

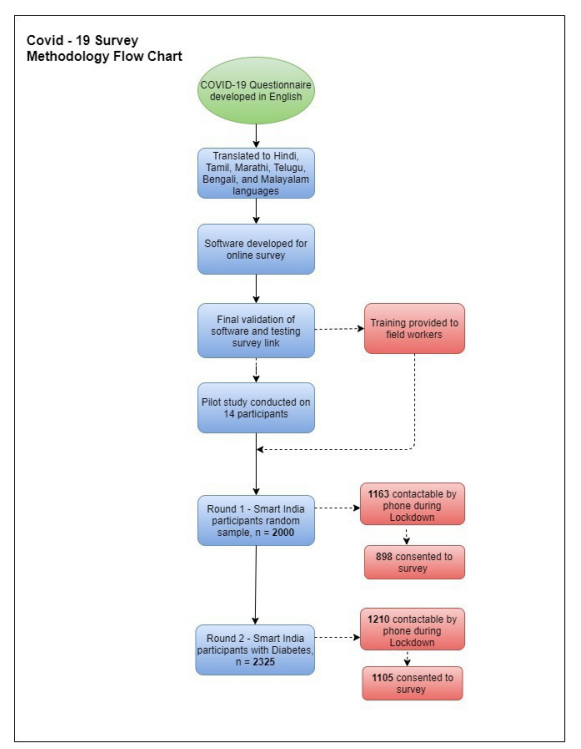

Figure 1 Flow diagram showing the derivation of the random samples.

data and estimated the difference in proportion of the outcome in more strict lockdown condition (red zone in lockdown phase 4) and less strict conditions. Proportion of times the difference was not significant (at $\alpha=0.05$ ) was considered as $\beta$, the probability of type II error and the power was then computed as $1-\beta$. We estimated the post hoc power for changes in all outcomes.

\section{Patient and public involvement statement}

Patients were not allowed to visit hospitals due to lockdown, so we assumed poor patient experiences based on the increased numbers of patients calling the hospitals for advice. Patients were not involved in the design of the study and were not involved in the recruitment to and conduct of the study. We intend to write a summary of the results to the participants who request for it.

\section{RESULTS}

The COVID-19 telephone survey was carried out on random samples of participants from the SMART India study cohort in two rounds, from 5 May to 24 May 2020 during phases 3 and 4 of the lockdown respectively. The derivation of the random samples from the SMART India cohort is shown in figure 1.The survey was conducted in the following states/union territories: Assam, Chhattisgarh, Karnataka, Kerala, Madhya Pradesh, Uttar Pradesh, Maharashtra, Odisha, Tamil Nadu, Telangana, West Bengal and Delhi. There was no change in zone classification between round 1 and 2 of the survey. A total of $2373 / 4325(55 \%)$ were contactable by telephone and 2003/2373 (84\%) consented and responded to the questionnaires.

The participant characteristics are shown in table 1 .

Nearly $83 \%$ of the sample were aged $30-65$ years, representing the population most likely be affected by the lockdown. More than half the sample were men and nearly 
Table 1 Participant characteristics and distribution among lockdown stages

\begin{tabular}{|c|c|c|c|c|c|c|}
\hline \multirow[b]{3}{*}{ Control variables } & \multirow[b]{3}{*}{$\mathrm{N}=2003(\%)$} & \multicolumn{4}{|c|}{ Lockdown stages (100\%) } & \\
\hline & & \multicolumn{2}{|l|}{ Three } & \multicolumn{2}{|l|}{ Four } & \\
\hline & & $\begin{array}{l}\text { Non-red } \\
\text { zone } \mathrm{N}=306\end{array}$ & $\begin{array}{l}\text { Red zone } \\
\mathrm{N}=592\end{array}$ & $\begin{array}{l}\text { Non-red } \\
\text { zone } \mathrm{N}=349\end{array}$ & $\begin{array}{l}\text { Red zone } \\
\mathrm{N}=756\end{array}$ & \\
\hline Age & & \multicolumn{4}{|c|}{$\begin{array}{l}\text { Weighted \% in the cross-table between lockdown } \\
\text { stages and control variables }\end{array}$} & \\
\hline $25-29$ years & $51(2.5)$ & 7.9 & 9 & 0 & 0 & \\
\hline$>65$ years & $284(14.2)$ & 1.7 & 2.5 & 2.4 & 4.1 & $\mathrm{p} \leq 0.001$ \\
\hline \multicolumn{7}{|l|}{ Gender } \\
\hline Male & $1098(54.8)$ & 7.8 & 19.4 & 7 & 16.5 & $F(1.93,3818.53)=2.675$ \\
\hline Female & $905(45.2)$ & 12.7 & 14 & 7.4 & 15.2 & $p=0.071$ \\
\hline Area & & 0 & 0 & 0 & 0 & \\
\hline Urban & $1516(75.7)$ & 17.5 & 23 & 11.9 & 24.1 & $F(1.88,3729.12)=4.040$ \\
\hline Rural & $487(24.3)$ & 3 & 10.3 & 2.5 & 7.6 & $p=0.020$ \\
\hline \multicolumn{7}{|l|}{ Living arrangements } \\
\hline Alone & $171(8.5)$ & 9.6 & 21.6 & 10 & 27.8 & \\
\hline With my spouse & $180(9.0)$ & 3 & 6.4 & 1.2 & 1.8 & \\
\hline $\begin{array}{l}\text { With my spouse, children } \\
\text { and others }\end{array}$ & $1561(77.9)$ & 1.1 & 4.7 & 1.3 & 2 & $F(4.08,8090.17)=15.361$ \\
\hline With non-related others & $91(4.5)$ & 6.8 & 0.7 & 1.9 & 0.1 & $\mathrm{p} \leq 0.001$ \\
\hline Absent & $746(37.2)$ & 18.3 & 29.5 & 0 & 0 & $F(2.06,4092.32)=423.311$ \\
\hline Present & 1257 (62.8) & 2.2 & 3.9 & 14.4 & 31.7 & $\mathrm{p}<0.001$ \\
\hline
\end{tabular}

$60 \%$ were working. Most of the respondents resided in urban areas and lived with family members. Approximately $60 \%$ of the participants described having some form of financial difficulties. As expected, $63 \%$ of the participants had diabetes

Table 1 also shows the weighted proportions of these variables adjusted for the complex design of the survey in cross-tabulation with the lockdown stages and the results of the tests for independence. In all cases except for gender and working the tests were highly significant. Therefore, in all models testing the association of outcomes with lockdown, these variables were entered as control variables.

\section{Associations of outcome measures with lockdown}

During the lockdown stages, in phase 3 and phase 4 , there were changes in the amount of restrictions imposed with some relaxation in the non-red zones, and the zones were based on the areas of varying severity of the COVID-19 pandemic, red zones being the worst affected. The crude associations between the lockdown stages and zones are presented in the table 2. As expected, negative outcomes were two to six times more likely in the more restrictive lockdown phase, and in areas with more severe impact of COVID-19. There were few exceptions. The discontinuity of care was found to be significantly less likely in the red zones (OR 0.62 [95\% CI 0.40 to 0.95]). There were no significant associations between non-availability of healthcare services and the phase of the lockdown, and poor health, and social loneliness with the severity of COVID-19 pandemic. However, when we considered the interaction between phases and zones, and adjusted for sociodemographic variables and presence of diabetes, the number of significant associations reduced suggesting the 


\begin{tabular}{|c|c|c|}
\hline \multirow[b]{2}{*}{ Lockdown stages } & \multicolumn{2}{|c|}{ Unadjusted OR $(95 \% \mathrm{Cl})$} \\
\hline & Four (ref: 3) & \\
\hline Zones & & Red (Ref: non-red) \\
\hline \multicolumn{3}{|l|}{ Healthcare variables } \\
\hline Non-availability & 1.17 (0.79 to 1.72$)$ & 2.61 (1.49 to 4.56$)$ \\
\hline Non-affordability & $1.93(1.44$ to 2.60$)$ & $2.22(1.47,3.35)$ \\
\hline Non-accessibility & 2.74 (2.03 to 3.70$)$ & 1.93 (1.26 to 2.94$)$ \\
\hline Inadequacy & 1.91 (1.42 to 2.57$)$ & 3.06 (1.97 to 4.74$)$ \\
\hline Inappropriate & 5.76 (4.29 to 7.72$)$ & 2.65 (1.81 to 3.88$)$ \\
\hline Discontinuity of care & 2.16 (1.49 to 3.12$)$ & $0.62(0.40$ to 0.95$)$ \\
\hline $\begin{array}{l}\text { Negative patient } \\
\text { experience }\end{array}$ & 0.75 (0.46 to 1.21$)$ & 4.91 (2.43 to 9.90$)$ \\
\hline \multicolumn{3}{|l|}{ Health and well-being } \\
\hline Poor health & 2.00 (1.36 to 2.94$)$ & 0.91 (0.53 to 1.57$)$ \\
\hline $\begin{array}{l}\text { WHO- } 5 \text { screening for } \\
\text { depression }\end{array}$ & 2.61 (1.99 to 3.42 ) & 2.99 (2.13 to 4.21$)$ \\
\hline Emotional loneliness & 1.70 (1.22 to 2.37$)$ & 4.35 (2.86 to 6.62$)$ \\
\hline Social loneliness & 2.57 (1.58 to 4.16$)$ & 1.16 (0.54 to 2.53$)$ \\
\hline $\begin{array}{l}\text { Loneliness } \\
\text { (emotional and/or } \\
\text { social) }\end{array}$ & 1.97 (1.06 to 3.65$)$ & 3.55 (1.62 to 7.81$)$ \\
\hline
\end{tabular}

complex nature of the relationships between our exposure variables and the outcomes.

Our main exposures of interest are the lockdown stages and zones. In tables 3 and 4 , we present the results for their main effects and interaction between them on healthcare provision and health and well-being. The main effect of the lockdown stage is the effect of lockdown stage 4 and non-red zones on the outcomes; the main effect of red zone is the effect of red zone and lockdown stage 3 on the outcomes; and the interaction effect is the effect of red zone and lockdown stage four on the outcomes. The main effects and interaction should be considered together when discussing the impact of COVID-19 lockdown on healthcare and health.

Except for healthcare availability and negative experience, on all other healthcare outcomes, the interaction between the zones and stages of lockdown had significant negative association (Non-availability: OR $0.626(95 \%$ CI 0.321 to 1.221 ), non-affordability: OR 1.917 (95\% CI 1.126 to 3.264 ), non-accessibility: OR 2.458 (95\% CI 1.549 to 3.902 ), inadequacy: OR 3.015 (95\% CI 1.616 to 5.625 ), inappropriateness: OR 2.225 (95\% CI 1.200 to 4.126), negative patient experience: OR 0.295 (95\% CI 0.069 to 1.264 ) and discontinuity of care: OR 6.756 [95\% CI 3.79 to 12.042]). This meant that in the most severe forms of lockdown, there was two to three times lack in healthcare service provision. In the earlier stage of lockdown, there was nearly four times reduction in the availability of healthcare services and six times more negative patient experience.
In the case of health and well-being outcomes, the lockdown related variables had fewer significant associations. The interaction between the zones and stages of lockdown was significantly associated with depression (OR 1.752 (95\% CI 1.065 to 2.882)) and social loneliness (OR 2.96 [95\% CI 1.306 to 6.709$]$ ).

Consistently, financial difficulties predicted negative outcomes in healthcare service provision as well as general, psychological and social well-being. On exploring further, we discovered that the effect of financial difficulties were considerably larger for three outcomes during the most severe lockdown compared with other times (non-accessibility: OR 11.81 (95\% CI 6.24 to 22.33 ) vs OR $1.82(95 \%$ CI 1.34 to 2.46$)$, relative difference (RD) $=6.5$; inappropriateness: OR 10.33 (95\% CI 2.95 to 36.21 ) vs OR 10.33 (95\% CI 2.95 to 36.21 ), $\mathrm{RD}=3.7$; discontinuity of care: OR 15.22 (95\% CI .58 to 30.57 ) vs OR $2.94(95 \%$ CI 2.08 to 4.15 ). See online supplemental table S2 for other results.

Post hoc sample size power analyses using simulation showed that the rejection rate with our sample size alpha level of 0.05 was $0 \%$ for all outcomes except for nonavailability $(8.4 \%)$, negative patient experience $(93.8 \%)$ and poor health $(8.1 \%)$.

\section{DISCUSSION}

Though lockdown was introduced as an urgent national mitigation strategy against COVID-19, our cross-sectional study has shown the degree to which healthcare provision and health and well-being of people were affected during lockdown in India, especially in the red zone.

The unadjusted associations in this study showed that lockdown affected access to healthcare and health and well-being of the participants negatively. However, multivariable logistic regression models revealed that the negative influences of the lockdown were mainly in areas of high burden of the pandemic. In contrast, availability of healthcare, negative patient experience and poor health were not associated with lockdown in these models. Among the health outcomes, mental health and loneliness were affected by the lockdown and the burden of pandemic while self-reported poor health had no impact. These results were similar to other studies from India. ${ }^{10} 18$ Verma and $\mathrm{Mishra}^{6}$ in their survey during the first lockdown in India reported the prevalence of moderate to severe depression, anxiety and stress to be $25 \%, 28 \%$ and $11.6 \%$, respectively, in the study participants.

However, the relation of the pandemic and lockdown is more complex. Our study supports previous reports that COVID-19 pandemic has resulted in some unexpected and sometimes beneficial outcomes due to changes in human behaviour and system responses. ${ }^{19}$ One such beneficial effect in our study was that the continuity of care was better in areas with greater impact of the COVID19. This could be the result on the focus on vulnerable population with pre-existing conditions. However, where the impact of the pandemic was higher and lockdown was 


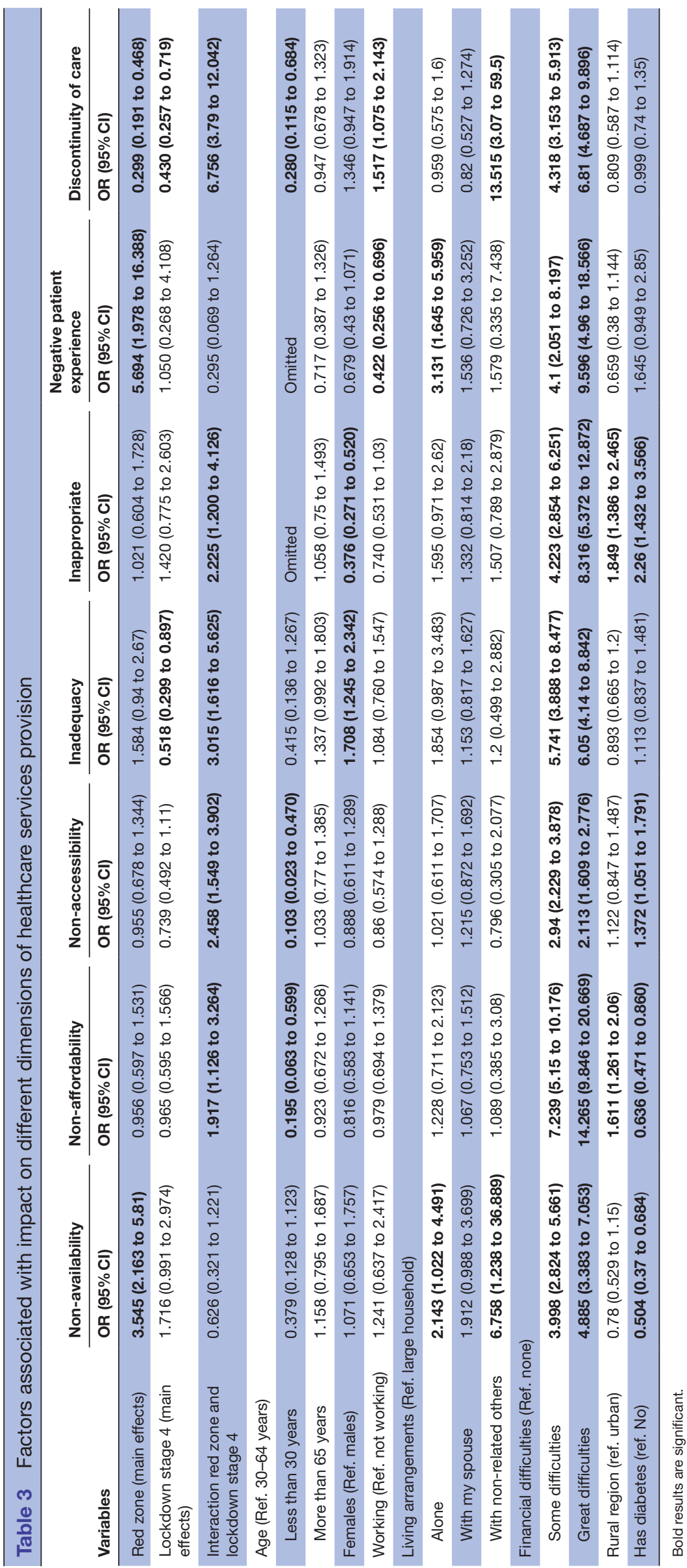




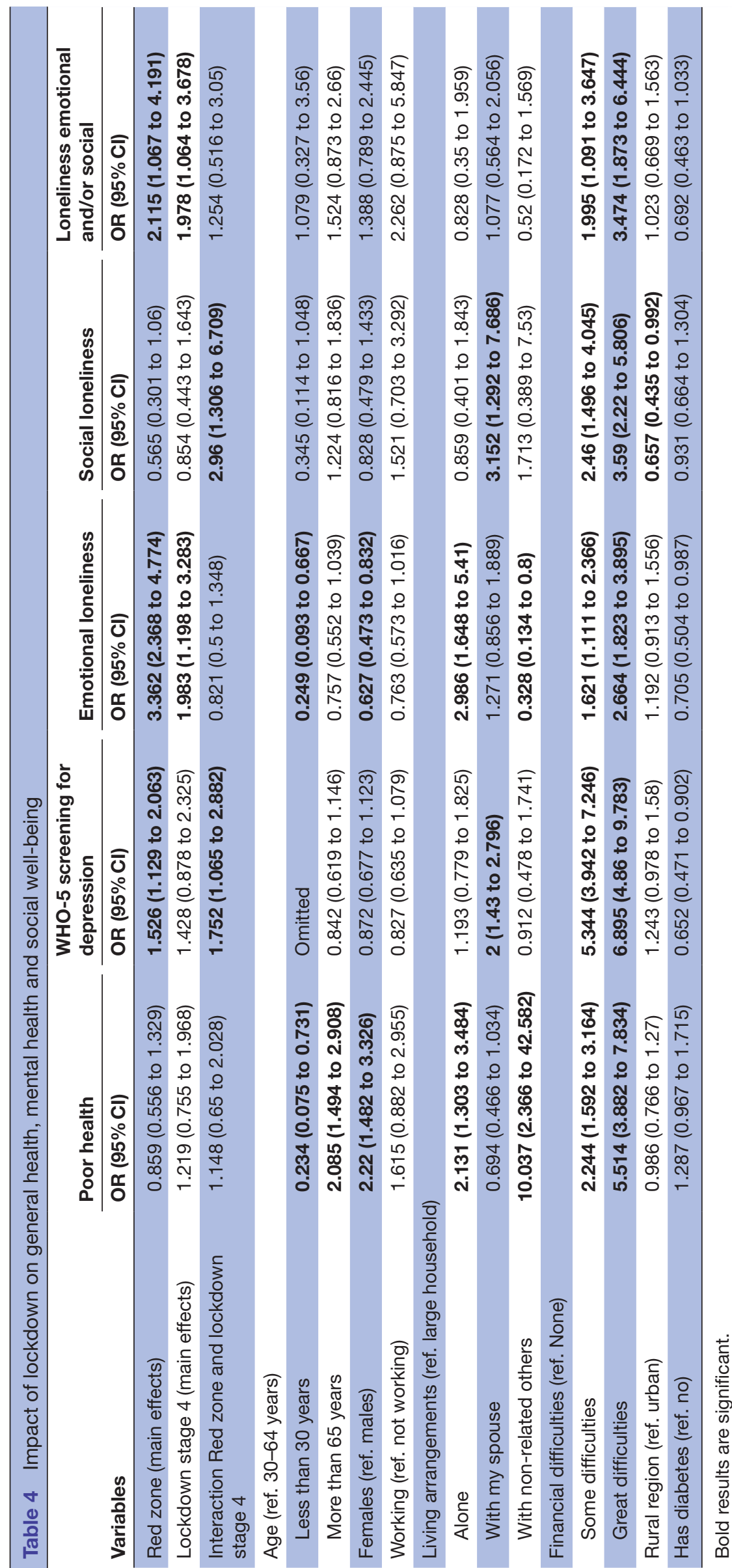


more restrictive, the healthcare outcomes were significantly poor with the exceptions of non-availability and negative patient experience. We cannot offer an explanation why more restrictive lockdown offered protection in these cases.

Several factors may account for the deteriorating mental health including the fear of COVID-19 infection, the fear of the unknown in terms of the length period of lockdown and the pandemic, consequences of the lockdown on finances and well-being, continuous exposure to reports on COVID-19 deaths worldwide and reduced access to extended family, friends and other social support systems. It is also interesting to note that social loneliness increased. This may represent the inability to cope with the restrictions despite the availability of internet and smartphone technology to stay connected.

We found the income inequality to be consistent across all outcomes with the likelihood of poor outcomes increasing as financial difficulties increased. Greater financial difficulties were associated with significantly greater odds of non-accessibility to healthcare (twelve times), inappropriate health provision (eleven times) and discontinuity of care (fifteen times). Greater financial stress also predicted poorer mental health, higher anxiety, depression and stress. It also modified the relationship between our main exposures and the outcomes (see online supplemental table S2). Historically pandemics accentuate the impact of socioeconomic inequalities and COVID-19 is no exception. ${ }^{20}{ }^{21}$ As a result, the impact of the COVID-19 pandemic will extend beyond the pandemic itself due to sustained and exaggerated inequalities, unless appropriate policy responses are made. The Government of India took a step in the right direction by reducing the financial burden on people such as an offer of optional moratorium on equated monthly instalments of all types of loans until the end of August 2020.

The need for social distancing, use of personal protection equipment, avoidance of surgery that may generate aerosols, testing of patients and staff and need for compulsory quarantine before a hospital admission have all caused significant constraints on the access, adequacy and appropriateness of healthcare services globally. These points were emphasised in the WHO report that stressed that the movement restriction measures of lockdown have considerable social and economic costs and should be implemented with the understanding, consent and participation of communities, and based on the principle of doing no harm. ${ }^{22}$ In India, Kerala's Abraham Committee report on post-lockdown planning also cautioned that the 'cure could be more expensive than the disease' and called for a graded approach to tackling the epidemic. ${ }^{23}$ Legislation of telemedicine is an important step in tackling the restrictions imposed on the current face to face consultations. The board of governors of Medical Council of India adopted the 'Telemedicine Practice Guidelines' which includes the overarching principles and practical framework of telemedicine for the country. In the wake of COVID-19 outbreak, it was amended and approved by the Ministry of Health and Family Welfare, Government of India officially on 25 March 2020. ${ }^{24}$ Thus, telehealth practices are adopted to a variable extend in the regions where the study was done. Although, most healthcare providers have rapidly switched to telephone and video consultations to keep in touch with their patients, our study shows that people do not perceive that postlockdown treatment they receive is appropriate compared with prelockdown. This requires a significant change in perceptions of both clinical staff and patients on adjusting to these uncertain times.

Our study results are in keeping with a recent predictive modelling on the impact of lockdown, Ghosal et al projected that the duration of lockdown is directly proportional to the worsening of glycaemic control and predicted an increase in diabetes-related complications with consequent additional load on an already overburdened public healthcare system. ${ }^{25}$ Another report indicated that the lockdown could cause weight gain due to poor physical activity, increased snacking and consumption of calorie-rich foods. ${ }^{26}$ On the contrary, a recent survey of people with type 2 diabetes in India showed better mean glycaemic control during lockdown compared with before lockdown. ${ }^{27}$ In our sensitivity analysis, we found that diabetes had no influence on the relationship between our outcomes and lockdown status. Although it as assumed that during the lockdown period, challenges in procuring regular prescribed medicines or lifestyle changes related to dietary habits, physical activity disruption could adversely impact people with diabetes, our results suggest that people with diabetes seem to cope with adversities of lockdown probably because they are already aware of available healthcare services. Indeed, the lockdown may have had a positive impact on some people, having more time to change to a healthier lifestyle.

The strengths of this study are that we were able to provide valuable information on the health impact during the COVID-19 lockdown in India. Because of our ongoing SMART India study, we were able to rapidly develop and deliver the questionnaire to participants who were accessible by telephone. The questionnaire was developed mainly from previously validated questionnaires. As far as we are aware, this is the first multicentre study in India conducted during the lockdown that evaluated the impact of the lockdown on healthcare services and general, social and mental health of the population. Our sample contained sufficient numbers of people with other known risk factors. About $20 \%$ of the sample were in the older age group. The sample also had adequate numbers of younger individuals to understand the impact in this group as recent data indicates that median age of COVID-19 infection in India is 38 years compared with the older age groups observed in the Western countries. ${ }^{6}$ Indians are among the ethnicities that face a statistically higher risk of death from COVID-19 compared with those of white ethnicity. ${ }^{28}$ From our models, we were also able to report that diabetes had no influence on the relationship between our outcomes and lockdown status. Our post 
hoc sample size calculation by simulation showed that the study had sufficient power for all the outcomes except the negative patient experience due to its low probability in the study.

Our study might be limited by its cross-sectional nature and use of participants of an existing study as the sampling frame. However, the existence of an accessible sample of randomly selected households across India was a motivating factor to undertake this study in the rapidly changing lockdown scenarios. Although our measures are from validated questionnaires, we acknowledge that these have never been piloted in India and so is a weakness of our study. Data were self-reported and not primary health or outcome data, the response rate was $50 \%$, so selection bias (even despite weighting) might have impacted the results and data were not collected prior to the lockdown or epidemic so making firm conclusions about its affect is difficult. Future studies are recommended to evaluate the impact of economic slowdown, unemployment and financial constraints in each household with time as people's responses focus on livelihood rather than lives.

\section{Author affiliations}

${ }^{1}$ Shri Bhagwan Mahavir Vitreoretinal Services, Medical Research Foundation,

Sankara Nethralaya, Chennai, India

${ }^{2}$ Dr.Mohan's Diabetes Specialities Centre and Madras Diabetes Research

Foundation, Chennai, India

${ }^{3}$ Diabetic Retinopathy Project, Sankara Nethralaya, Chennai, India

${ }^{4}$ UCL Institute of Ophthalmology, London, UK

${ }^{5}$ NIHR Moorfields Biomedical Research Centre, Moorfields Eye Hosp NHS Foundation Trust, London, UK

${ }^{6}$ B005 Meenakshi Classic, Bangalore, India

${ }^{7}$ Madras Diabetes Research Foundation, Chennai, India

${ }^{8}$ University of East London, London, UK

Acknowledgements SMART India study Group Investigators. We also would like to thank our reviewers, Mark Siedner and Pragati B Hebbar for providing useful input into strengthening this manuscript.

Contributors RRaman, RRaj, SS, DC and GN contributed in literature search, study design, data interpretation. RRaman, RRaj, SS, RRam and DC helped in writing the manuscript. JS, RRaman, RRaj, RRam, GN helped in data collection. SS, DC and GN contributed in data analysis and making figures. JPT helped in study design, data collection. VM contributed in study design, writing.

Funding This work was supported by SMART India study, part of the ORNATE India project, is funded by the GCRF UKRI (MR/P207881/1). The research is supported by the NIHR Biomedical Research Centre at Moorfields Eye Hospital NHS Foundation Trust and UCL Institute of Ophthalmology, London, UK.

Competing interests None declared.

Patient consent for publication Not required.

Ethics approval The Institutional Ethics Committee of Vision Research Foundation, Sankara Nethralaya, Chennai, India approved the study (VRF/674A-2018-P).

Provenance and peer review Not commissioned; externally peer reviewed.

Data availability statement Data are available on reasonable request. The datasets generated during and/or analysed during the current study are not publicly available, as it is against the organisation/hospital policy. But are available from the corresponding author on reasonable request.

Supplemental material This content has been supplied by the author(s). It has not been vetted by BMJ Publishing Group Limited (BMJ) and may not have been peer-reviewed. Any opinions or recommendations discussed are solely those of the author(s) and are not endorsed by BMJ. BMJ disclaims all liability and responsibility arising from any reliance placed on the content. Where the content includes any translated material, BMJ does not warrant the accuracy and reliability of the translations (including but not limited to local regulations, clinical guidelines, terminology, drug names and drug dosages), and is not responsible for any error and/or omissions arising from translation and adaptation or otherwise.

Open access This is an open access article distributed in accordance with the Creative Commons Attribution Non Commercial (CC BY-NC 4.0) license, which permits others to distribute, remix, adapt, build upon this work non-commercially, and license their derivative works on different terms, provided the original work is properly cited, appropriate credit is given, any changes made indicated, and the use is non-commercial. See: http://creativecommons.org/licenses/by-nc/4.0/.

ORCID iD

Rajiv Raman http://orcid.org/0000-0001-5842-0233

\section{REFERENCES}

1 Ferguson N, Laydon D, Nedjati-Gilani G. Impact of nonpharmaceutical interventions (NPIs) to reduce COVID-19 mortality and healthcare demand. Imperial College of London.

2 WHO. World Health Organization novel coronavirus (2019-nCoV) situation report - 11, 2019. Available: https://www.who.int/ emergencies/diseases/novel-coronavirus-2019/situation-reports [Accessed 9 Jun 2020].

3 Remuzzi A, Remuzzi G. COVID-19 and Italy: what next? Lancet 2020;395:1225-8.

4 Mazumder A, Arora M, Bharadiya V, et al. SARS-CoV-2 epidemic in India: epidemiological features and in silico analysis of the effect of interventions. F1000Res 2020;9:315.

5 Bhattacharjee A, Kumar M, Patel KK. When COVID-19 will decline in India? prediction by combination of recovery and case load rate. Clin Epidemiol Glob Health 2021;9:17-20.

6 Verma S, Mishra A. Depression, anxiety, and stress and sociodemographic correlates among general Indian public during COVID-19. Int J Soc Psychiatry 2020;66:756-62.

7 Sivaprasad S, Raman R, Conroy D, et al. The ORNATE India project: United Kingdom-India research collaboration to tackle visual impairment due to diabetic retinopathy. Eye 2020;34:1279-86.

8 COVID-19: Lockdown across India, in line with WHO guidance. UN News. 24 Mar 2020.

9 Gulliford M, Figueroa-Munoz J, Morgan M, et al. What does 'access to health care' mean? J Health Serv Res Policy 2002;7:186-8.

10 Penchansky R, Thomas JW. The concept of access: definition and relationship to consumer satisfaction. Med Care 1981;19:127-40.

11 European Patients Forum. Access to health care, 2016. Available: https://www.eu-patient.eu/globalassets/policy/access/final-accesssurvey-report_16-dec.pdf [Accessed 12 Jun 2020].

12 Webster TR, Mantopoulos J, Jackson E, et al. A brief questionnaire for assessing patient healthcare experiences in low-income settings. Int J Qual Health Care 2011;23:258-68.

13 Freeman GK, Olesen F, Hjortdahl P. Continuity of care: an essential element of modern general practice? Fam Pract 2003;20:623-7.

14 Naithani S, Gulliford M, Morgan M. Patients' perceptions and experiences of 'continuity of care' in diabetes. Health Expect 2006;9:118-29.

15 Topp CW, Østergaard SD, Søndergaard S, et al. The WHO-5 well-being index: a systematic review of the literature. Psychother Psychosom 2015;84:167-76.

16 De Jong Gierveld J, Van Tilburg T. A six-item scale for overall, emotional and social loneliness: confirmative tests on new survey data. Res Aging 2006;28:582-98.

17 Weiss RS. Loneliness: the experience of emotional and social isolation. Cambridge, MA: MIT Press, 1973.

18 Rehman U, Shahnawaz MG, Khan NH, et al. Depression, anxiety and stress among Indians in times of Covid-19 Lockdown. Community Ment Health J 2020;7309680 doi:10.1007/s10597-020-00664-x

19 Nelson B. The positive effects of covid-19. BMJ 2020;369:m1785.

20 Kawachi I. COVID-19 and the 'rediscovery' of health inequities. Int $J$ Epidemiol 2020;49:1415-8.

21 Bambra C, Riordan R, Ford J, et al. The COVID-19 pandemic and health inequalities. J Epidemiol Community Health 2020;74:964.

22 WHO. COVID-19 strategy update. Geneva: World Health Organization, 2020. https://www.who.int/publications/i/item/covid19-strategy-update-14-april-2020

23 Government of Kerala. Report of the expert committee on strategy for easing lockdown restrictions. Thiruvananthapuram, 2020. Available: https://www.pslhub.org/learn/coronavirus-COVID-19/ lockdown-exit-strategies/government-of-kerala-covid-19-report-ofthe-expert-committee-on-strategy-for-easing-lockdown-restrictions6-april-2020-r2220/ 
24 Telemedicine Practice Guidelines - Enabling Registered Medical Practitioners to Provide Healthcare Using Telemedicine. Appendix 5 of the Indian medical council (professional conduct, etiquette and ethics regulation), 2002. Available: https://www.mohfw.gov.in/pdf/ Telemedicine.pdf

25 Ghosal S, Sinha B, Majumder M. Estimation of effects of nationwidelockdown for containing coronavirus infection on worsening of glycosylatedhaemoglobin and increase in diabetesrelated complications: a simulationmodel using multivariate regression analysis. Diabetes MetabSyndr 2020;14:319-23.
26 Ghosh A, Arora B, Gupta R, et al. Effects of nationwide lockdown during COVID-19 epidemic on lifestyle and other medical issues of patients with type 2 diabetes in North India. Diabetes Metab Syndr 2020;14:917-20.

27 Anjana RM, Pradeepa R, Deepa M. Acceptability and utilisation of technologies and effect of glycemic control in type 2 diabeteslessons learnt from lockdown. Diabetes TechnolTher 2020.

28 Khunti K, Singh AK, Pareek M, et al. Is ethnicity linked to incidence or outcomes of COVID-19? BMJ 2020;369:m1548. 